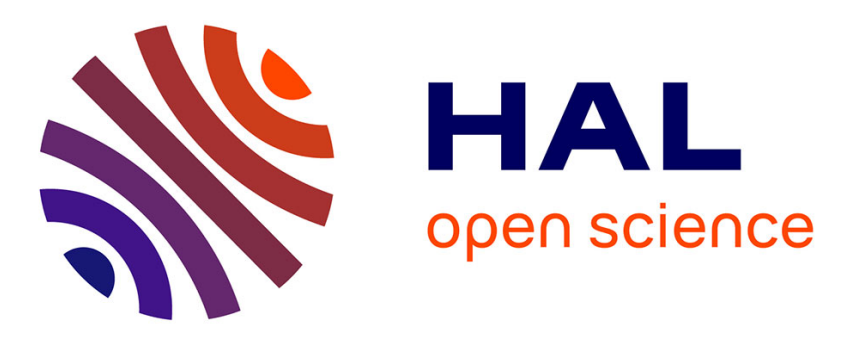

\title{
Cyclotomic Units and Class Groups in Z_p-extensions of real abelian number fields
}

Filippo Alberto Edoardo Nuccio Mortarino Majno Di Capriglio

\section{To cite this version:}

Filippo Alberto Edoardo Nuccio Mortarino Majno Di Capriglio. Cyclotomic Units and Class Groups in Z_p-extensions of real abelian number fields. Mathematical Proceedings of the Cambridge Philosophical Society, 2009, 148, pp.93-106. 10.1017/S0305004109990119 . hal-00947151

\section{HAL Id: hal-00947151 \\ https://hal.science/hal-00947151}

Submitted on 21 Feb 2014

HAL is a multi-disciplinary open access archive for the deposit and dissemination of scientific research documents, whether they are published or not. The documents may come from teaching and research institutions in France or abroad, or from public or private research centers.
L'archive ouverte pluridisciplinaire HAL, est destinée au dépôt et à la diffusion de documents scientifiques de niveau recherche, publiés ou non, émanant des établissements d'enseignement et de recherche français ou étrangers, des laboratoires publics ou privés. 


\title{
Cyclotomic units and class groups in $\mathbb{Z}_{p}$-extensions of real abelian fields
}

\author{
BY FILIPPO ALBERTO EDOARDO NUCCIO \\ Università “La Sapienza”, \\ P.le Aldo Moro, 5-00186, Rome, Italy. \\ e-mail: nuccio@mat.uniroma1.it
}

(Received 9 December 2008; revised 24 April 2009)

\begin{abstract}
For a real abelian number field $F$ and for a prime $p$ we study the relation between the $p$-parts of the class groups and of the quotients of global units modulo cyclotomic units along the cyclotomic $\mathbb{Z}_{p}$-extension of $F$. Assuming Greenberg's conjecture about the vanishing of the $\lambda$-invariant of the extension, a map between these groups has been constructed by several authors, and shown to be an isomorphism if $p$ does not split in $F$. We focus in the split case, showing that there are, in general, non-trivial kernels and cokernels.
\end{abstract}

\section{Introduction}

Let $F / \mathbb{Q}$ be a real abelian field of conductor $f$ and let $C l_{F}$ be its ideal class group. A beautiful formula for the order of this class group comes from the group of cyclotomic units: this is a subgroup of the global units $\mathcal{O}_{F}^{\times}$whose index is linked to the order of $C l_{F}$. To be precise, we give the following definition ([Sin81, section 4]):

Definition 1.1. For integers $n>1$ and $a$ not divisible by $n$, let $\zeta_{n}$ be a primitive $n$th root of unity. Then $N \operatorname{orm}_{F \cap \mathbb{Q}\left(\zeta_{n}\right)}^{\mathbb{Q}\left(\zeta_{n}\right)}\left(1-\zeta_{n}^{a}\right) \in F$ and we define the cyclotomic numbers $D_{F}$ to be the subgroup of $F^{\times}$generated by -1 and $\operatorname{Norm}_{F \cap \mathbb{Q}\left(\zeta_{n}\right)}^{\mathbb{Q}\left(\zeta_{n}\right)}\left(1-\zeta_{n}^{a}\right)$ for all $n>1$ and all $a$ not divisible by $n$. Then we define the cyclotomic units of $F$ to be

$$
C y c_{F}:=D_{F} \cap \mathcal{O}_{F}^{\times}
$$

Sinnott proved in [Sin81, theorem $4 \cdot 1$, with proposition 5.1], the following theorem:

THEOREM (Sinnott). There exists an explicit constant $\kappa_{F}$ divisible only by 2 and by primes dividing $[F: \mathbb{Q}]$ such that

$$
\left[\mathcal{O}_{F}^{\times}: C y c_{F}\right]=\kappa_{F}\left|C l_{F}\right|
$$

Let now $p$ be an odd prime that does not divide $[F: \mathbb{Q}]$ : by tensoring $\mathcal{O}_{F}^{\times}, C y c_{F}$ and $C l_{F}$ with $\mathbb{Z}_{p}$ we get an equality

$$
\left[\mathcal{O}_{F}^{\times} \otimes \mathbb{Z}_{p}: C y c_{F} \otimes \mathbb{Z}_{p}\right]=\left|C l_{F} \otimes \mathbb{Z}_{p}\right|
$$

and it is natural to ask for an algebraic interpretation of this. Moreover, observe that our assumption $p \nmid[F: \mathbb{Q}]$ makes the Galois group $\Delta:=\operatorname{Gal}(F / \mathbb{Q})$ act on the modules appearing above through one-dimensional characters, and we can decompose them accordingly: in the sequel we write $M(\chi)$ for every $\mathbb{Z}[\Delta]$-module $M$ to mean the submodule of 
$M \otimes \mathbb{Z}_{p}[\operatorname{Im}(\chi)]$ on which $\Delta$ acts as $\chi$, where $\chi \in \hat{\Delta}$ (see the end of Section 2 for a precise discussion). Then an even more optimistic question is to hope for a character-by-character version of Sinnott's theorem, namely

$$
\left[\mathcal{O}_{F}^{\times}(\chi): C y c_{F}(\chi)\right] \stackrel{?}{=}\left|C l_{F}(\chi)\right|
$$

and then ask for an algebraic interpretation of this. Although it is easy to see that these $\Delta$-modules are in general not isomorphic (see the example on page 143 of [KS95]), it can be shown that they sit in an exact sequence for a wide class of fields arising in classical Iwasawa theory. More precisely, let $F_{\infty} / F$ be the cyclotomic $\mathbb{Z}_{p}$-extension of $F$ and let $\Gamma=\operatorname{Gal}\left(F_{\infty} / F\right) \cong \mathbb{Z}_{p}$ : then

$$
F_{\infty}=\bigcup_{n \geqslant 0} F_{n} \supset \cdots \supset F_{n} \supset F_{n-1} \supset \cdots \supset F_{0}=F
$$

where $F_{n} / F$ is a cyclic extension of degree $p^{n}$ whose Galois group is isomorphic to $\Gamma / \Gamma^{p^{n}}$. In a celebrated work (see [Iwa73]) Iwasawa gives a formula for the growth of the order of $C l_{F_{n}} \otimes \mathbb{Z}_{p}$ : he proves that there are three integers $\mu, \lambda$ and $\nu$, and an index $n_{0} \geqslant 0$, such that

$$
\left|C l_{F_{n}} \otimes \mathbb{Z}_{p}\right|=p^{\mu p^{n}+\lambda n+v} \text { for every } n \geqslant n_{0} .
$$

Moreover, Ferrero and Washington proved in [FW79] that the invariant $\mu$ vanishes. A longstanding conjecture by Greenberg (see [Gre76], where conditions for this vanishing are studied) predicts that $\lambda=0$ : according to the conjecture the $p$-part of the class groups should stay bounded in the tower.

Although a proof of this conjecture has never been provided, many computational checks have been performed verifying the conjecture in many cases (see, for instance, [KS95]). Under the assumptions $\lambda=0$ and $\chi(p) \neq 1$, i. e. $p$ does not split in $F$, some authors (see [BNQD01], [KS95], [Kuz96] and [Oza97]) were able to construct an explicit isomorphism

$$
\alpha: C l_{F_{n}}(\chi) \cong\left(\mathcal{O}_{F_{n}}^{\times} / C y c_{F_{n}}\right)(\chi)
$$

if $n$ is big enough. Although the construction of the above morphism works also in the case $\chi(p)=1$, as detailed in the beginning of Section 5, the split case seems to have never been addressed. We focus then on this case, and study the map in this context, still calling it $\alpha$. Our main result is the following (see Corollary 5.2):

THEOREM. With notations as above, assume that $\chi$ is a non-trivial character of $\Delta$ such that $\chi(p)=1$ and that $\lambda=0$. Then, for sufficiently big $n$, there is an exact sequence

$$
0 \longrightarrow \mathrm{K} \longrightarrow C l_{F_{n}}(\chi) \stackrel{\alpha}{\longrightarrow}\left(\mathcal{O}_{F_{n}}^{\times} / C y c_{F_{n}}\right)(\chi) \longrightarrow \mathrm{C} \longrightarrow 0:
$$

both the kernel $\mathrm{K}$ and the cokernel $\mathrm{C}$ of $\alpha$ are finite groups with trivial $\Gamma$-action isomorphic to $\mathbb{Z}_{p}[\operatorname{Im}(\chi)] / L_{p}(1, \chi)$ where $L_{p}(s, \chi)$ is the Kubota-Leopoldt p-adic L-function.

\section{Some Tate cohomology}

In this section we briefly recall some well-known facts that are useful in the sequel. Throughout, $L / K$ is a cyclic extension of number fields, whose Galois group we denote by $G$. In our application, $K$ and $L$ will usually be layers $F_{m}$ and $F_{n}$ of the cyclotomic $\mathbb{Z}_{p^{-}}$ extension for some $n \geqslant m$, but we prefer here not to restrict to this special case. 
We need to introduce some notation. Let

$$
\mathbb{U}_{K}=\prod_{v \nmid \infty} \mathcal{O}_{K, v}^{\times} \times \prod_{v \mid \infty} K_{v}^{\times}
$$

be the idèle units, $i$. $e$. idèles having valuation 0 at all finite place $v$ (we refer the reader to sections $14-19$ of Cassels' paper in [CF86] for basic properties of idèles and idèle class group) and let $\Sigma$ be the set of places of $K$ that ramify in $L / K$. It is known (see [CF86, Serre, section 1.4]) that the Tate cohomology of local units in an unramified extension of local fields is trivial: therefore Shapiro's Lemma ([CF86, Tate, proposition 7.2]) gives

$$
\hat{H}^{q}\left(G, \mathbb{U}_{L}\right)=\hat{H}^{q}\left(G, \prod_{v \in \Sigma} \prod_{w \mid v} \mathcal{O}_{L, w}^{\times}\right) \cong \prod_{v \in \Sigma} \hat{H}^{q}\left(G_{v}, \mathcal{O}_{L, w}^{\times}\right),
$$

where we fix a choice of a place $w$ of $L$ above $v$ for every $v \in \Sigma$ and we denote by $G_{v}$ its decomposition group in $G$; we will make this identification throughout. We denote the product of local units at places $v \in \Sigma$ appearing above by $U_{\Sigma}$. Consider the following commutative diagram of $G$-modules:

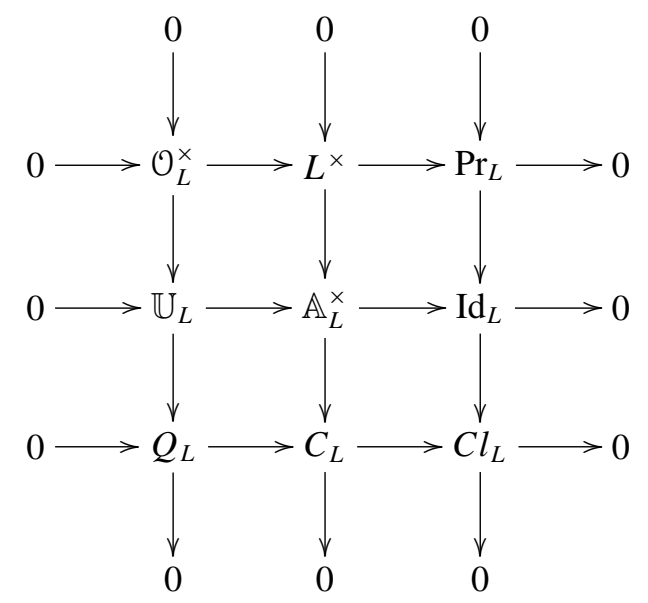

Here $\operatorname{Id}_{L}$ and $\operatorname{Pr}_{L}$ denote the group of all fractional ideals of $L$ and of principal ideals, respectively; while $C_{L}=\mathbb{A}_{L}^{\times} / L^{\times}$is the group of idèle classes and $Q_{L}=\mathbb{U}_{L} / \mathcal{O}_{L}^{\times}$.

LEMMA 2.1. Taking Tate cohomology of (2.1) and exploiting the fact that $G$ is cyclic, we find the diagram:

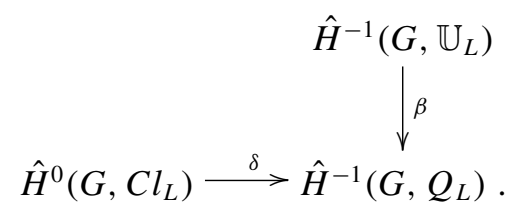

Then $\operatorname{Im}(\beta)$ is generated by the image via $\delta$ of classes $\left[\operatorname{Norm}_{G / G_{v}}\left(\mathrm{P}_{v}\right)\right]$ for $\mathrm{P}_{v}$ running through all primes in L above $\Sigma$.

Proof. First of all, the above decomposition

$$
\hat{H}^{-1}\left(G, \mathbb{U}_{L}\right) \cong \prod_{v \in \Sigma} \hat{H}^{-1}\left(G_{v}, \mathcal{O}_{w}^{\times}\right)
$$

allows to write $\beta=\prod \beta_{v}$ where $\beta_{v}$ is the restriction of $\beta$ to the $v$ th factor $\hat{H}^{-1}\left(G_{v}, \mathcal{O}_{w}^{\times}\right)$. 
We therefore fix a place $v \in \Sigma$ and we show that $\operatorname{Im}\left(\beta_{v}\right)$ is the class of $\delta\left(\sum_{\sigma \in G / G_{v}} \sigma \mathfrak{P}_{v}\right)$ for some prime $\mathfrak{P}_{v}$ of $L$ above $v$; observe that the sum (written by a small abuse of notation, since we are summing over representatives of $G / G_{v}$ in $G$ - but their choice is harmless) makes sense, since $\mathfrak{P}_{v}$ is fixed by $G_{v}$. Choosing a uniformizer $\pi_{w}$ at $\mathfrak{P}_{w}$ we get a generator $(\tau-1) \pi_{w}$ of $\hat{H}^{-1}\left(G_{v}, \mathcal{O}_{w}^{\times}\right)$, where $\tau$ is a generator of $G_{v}$ : the isomorphism of Shapiro's Lemma gives

$$
\begin{aligned}
\hat{H}^{-1}\left(G_{v}, \mathcal{O}_{w}^{\times}\right) \stackrel{\cong}{\longrightarrow} \prod_{w \mid v} \hat{H}^{-1}\left(G, \mathcal{O}_{w}^{\times}\right) \\
(\tau-1) \pi_{w} \longmapsto\left\{\sigma\left((\tau-1) \pi_{w}\right)\right\}_{\sigma \in G / G_{v}}
\end{aligned}
$$

and therefore the image of $\beta_{v}$ is generated by $\sum_{\sigma \in G / G_{v}} \sigma\left((\tau-1) \pi_{w}\right):=\vartheta_{v}$.

On the other hand, the map $\delta$ is given (see [NSW00, chapter I, section 2]) by $x \mapsto(\gamma-1) x$, where $\gamma$ is a generator of $G$, for all $x \in \hat{H}^{0}\left(G, C l_{L}\right)$. As $C l_{L}=$ $\mathrm{Id}_{L} / \operatorname{Pr}_{L} \cong C_{L} / Q_{L}$, we can write the class $\left[\mathfrak{P}_{w}\right] \in C l_{L}$ as $\left[\left(\ldots, 1, \pi_{w}, 1, \ldots\right)\right]$ where $\left(\ldots, 1, \pi_{w}, 1, \ldots\right) \in \mathbb{A}_{L}^{\times}$is the idèle whose $w$-component is $\pi_{w}$ and all other components are 1. Then $\operatorname{Norm}_{G / G_{v}}\left[\mathfrak{P}_{w}\right]=\sum_{\sigma \in G / G_{v}} \sigma\left[\left(\ldots, 1, \pi_{w}, 1, \ldots\right)\right] \in H^{0}\left(G, C l_{L}\right)$ and the image via $\delta$ of its class in $\hat{H}^{0}\left(G, C l_{L}\right)$ is clearly $\vartheta_{v}$ (observe that $\sum_{\sigma} \sigma(\tau-1)=\sum_{\sigma} \sigma(\gamma-1)$ ).

PROPOSITION 2.2 (See [Iwa73]). Let $\mathrm{J}: C l_{K} \rightarrow C l_{L}$ be the map induced by extending fractional ideals of $\mathcal{O}_{K}$ to $\mathcal{O}_{L}$. Then

$$
\operatorname{Ker}(\jmath) \cong \operatorname{Ker}\left(\hat{H}^{1}\left(G, \mathcal{O}_{L}^{\times}\right) \longrightarrow \hat{H}^{1}\left(G, U_{\Sigma}\right)\right) .
$$

Proof. We simply apply the Snake Lemma twice. First of all, apply it to

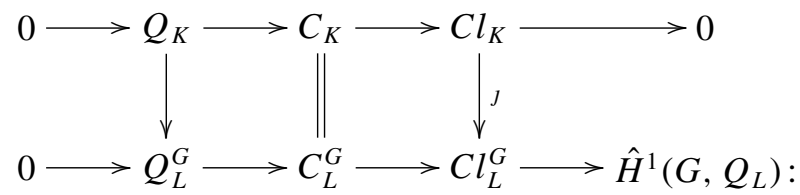

it shows $\operatorname{Ker}(\jmath) \cong Q_{L}^{G} / Q_{K}$. Then apply it to

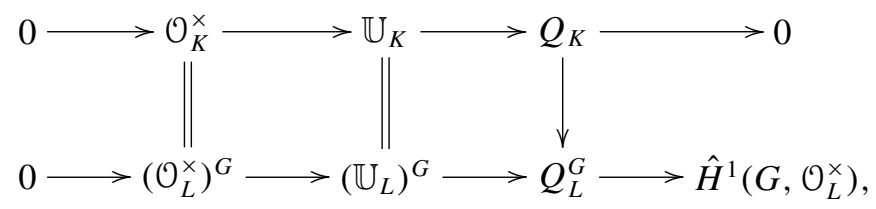

finding $Q_{L}^{G} / Q_{K} \cong \operatorname{Ker}\left(\hat{H}^{1}\left(G, \mathcal{O}_{L}^{\times}\right) \rightarrow \hat{H}^{1}\left(G, U_{\Sigma}\right)\right)$.

Remark. The above proof does not use the hypothesis that $G$ be cyclic. In fact, we will in the sequel apply the proposition assuming this cyclicity, finding $\operatorname{Ker}(J) \cong$ $\operatorname{Ker}\left(\hat{H}^{-1}\left(G, \mathcal{O}_{L}^{\times}\right) \rightarrow \hat{H}^{-1}\left(G, U_{\Sigma}\right)\right)$. We remark that in his thesis [Gre76] Greenberg gives the following criterion: $\mu=\lambda=0$ if and only if for all $m \geqslant 0$ the map $j$ relative to the cyclic extension $F_{n} / F_{m}$, restricted to $p$-Sylow subgroups, becomes 0 for sufficiently large $n$ (possibly depending on $m$ ). This will be the starting point for our proof of Theorem $5 \cdot 1$.

We end this section with some remarks on the action of $\Delta$ on the cohomology along a $\mathbb{Z}_{p}$-extension. Pick a $p$-adic character $\chi$ of $\Delta$; setting $R_{\chi}=\mathbb{Z}_{p}[\operatorname{Im}(\chi)]$, we let $\delta \in \Delta$ act on 
$R_{\chi}$ by $x \mapsto \chi(\delta) x$. In this way, $R_{\chi}$ becomes a $\mathbb{Z}_{p}[\Delta]$-algebra. For every $\mathbb{Z}[\Delta]$-module $M$ we denote by $M(\chi)$ the $\chi$-eigenspace of $M \otimes_{\mathbb{Z}_{p}[\Delta]} R_{\chi}$ for the action of $\Delta:$ it is defined as $M(\chi)=\left\{m \in M \otimes R_{\chi}\right.$ s.t. $\left.\delta \cdot m=\chi(\delta) \cdot m \forall \delta \in \Delta\right\} \cong \operatorname{Hom}_{\mathbb{Z}_{p}[\Delta]}\left(R_{\chi}, M \otimes \mathbb{Z}_{p}\right)$ : for more details, see [Sch88, section 2]. Then we have the following

LEMMA 2.3 [Sch88, lemma 2.1]. Suppose that $G$ and $\Delta$ are abelian groups such that $p \nmid|\Delta|$ and $G$ is a p-group. Let $M$ be $a \mathbb{Z}[\Delta \times G]$-module, then:

(i) for every $q \in \mathbb{Z}$, the natural map

$$
\hat{H}^{q}\left(G, M \otimes \mathbb{Z}_{p}\right)^{\Delta} \longrightarrow \hat{H}^{q}\left(G,\left(M \otimes \mathbb{Z}_{p}\right)^{\Delta}\right)
$$

is an isomorphism (of abelian groups with trivial $\Delta \times G$-action)

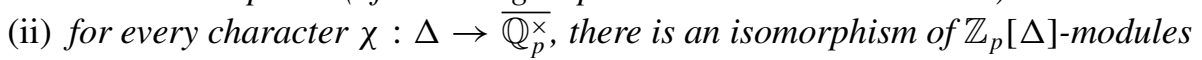

$$
\hat{H}^{q}(G, M(\chi)) \cong \hat{H}^{q}(G, M)(\chi) \quad \forall q \in \mathbb{Z} .
$$

\section{Cyclotomic units}

We now go back to the situation described in the introduction: let then $F_{\infty} / F$ be the $\mathbb{Z}_{p^{-}}$ extension of $F$ and denote by $\Gamma$ its Galois group. For every $n$ and for every prime ideal $\mathrm{p} \subseteq \mathcal{O}_{F_{n}}$ dividing $p$, we let $\mathcal{O}_{F_{n}, \mathrm{p}}^{\times}$denote the local units at p. Also, we let $C y c_{F_{n}}$ be the cyclotomic units of $F_{n}$ as defined by Sinnott in [Sin81]. Fix from now on a non-trivial character $\chi \neq 1$ of $\Delta$ and an odd prime $p \nmid[F: \mathbb{Q}]$ such that $\chi(p)=1$ : accordingly, let $R_{\chi}=\mathbb{Z}_{p}[(\operatorname{Im}(\chi)]$, seen as a $\Delta$-module as detailed at the end of Section 2. Remark that for every $n \geqslant 0, \operatorname{Gal}\left(F_{n} / \mathbb{Q}\right) \cong \Delta \times \operatorname{Gal}\left(F_{n} / F\right)$, and this turns $F_{n}^{\times}, C y c_{F_{n}}, C l_{F_{n}}$, etc ...into $\Delta$-modules.

Consider a contravariant functor

$$
\mathscr{M}: \underline{\operatorname{SGr}(\Delta)} \longrightarrow \underline{\mathbb{Z}[\Delta]-\operatorname{Mod}}
$$

from the category of subgroups of $\Delta$ (morphisms being inclusions) to that of $\mathbb{Z}[\Delta]$-modules, verifying the condition (compare with [NSW00])

$$
\mathscr{M}(H) \cong \mathscr{M}(1)^{H} \text { for all } H \triangleleft \Delta \text { functorially in } H .
$$

Example. Prototypical examples of such a functor are the additive group $\mathscr{M}(H):=$ $F_{n}^{H}$, or the global (resp. cyclotomic, local) units $\mathscr{M}(H):=O_{F_{n}^{H}}^{\times}(\operatorname{resp} . \mathscr{M}(H):=$ $\left.C y c_{F_{n}^{H}}, \mathscr{M}(H)=\mathcal{O}_{F_{n}^{H}, \mathrm{p}}^{\times}\right)$, together with the natural inclusions $F_{n}^{H} \stackrel{F^{n}}{\hookrightarrow} F_{n}, \mathcal{O}_{F_{n}^{H}}^{\times} \hookrightarrow \mathcal{O}_{F_{n}}^{\times}$, ecc... Moreover, since $p \nmid|\Delta|$, the $p$-part of the class group verifies (3.1): indeed, for all $H$ the map $C l_{F_{n}^{H}} \stackrel{J}{\rightarrow} C l_{F_{n}}^{H} \stackrel{\text { Norm }}{\rightarrow} C l_{F_{n}^{H}}$ coincides with the multiplication by $[\Delta: H]$, and is therefore an isomorphism when restricted to $p$-Sylow (and this for all $n \geqslant 0$ ). Similarly, we have $H^{1}\left(H, C y c_{n} \otimes \mathbb{Z}_{p}\right)=0$ for all $H \triangleleft \Delta$ because $p \nmid|H|$ (apply Corollary 1 to Proposition 8 of Atiyah and Wall's paper in [CF86]), so

$$
\left(\left(\mathcal{O}_{F_{n}}^{\times} / C y c_{F_{n}}\right) \otimes \mathbb{Z}_{p}\right)^{H} \cong\left(\mathcal{O}_{F_{n}^{H}} / C y c_{F_{n}^{H}}\right) \otimes \mathbb{Z}_{p}
$$

and also the functors $\mathscr{M}(H)=\left(\mathcal{O}_{F_{n}^{H}} / C y c_{F_{n}^{H}}\right) \otimes \mathbb{Z}_{p}$ verify condition (3.1) for all $n \geqslant 0$.

Suppose now that a functor as above is given, and that it verifies $(3 \cdot 1)$ : then for every character $\chi$ of $\Delta$, there is a natural identification $\mathscr{M}(1)(\chi) \cong \mathscr{M}(\operatorname{Ker}(\chi))(\chi)$. Indeed, $\mathscr{M}(\operatorname{Ker}(\chi)) \cong \mathscr{M}(1)^{\operatorname{Ker}(\chi)} \subseteq \mathscr{M}(1)$, while $\mathscr{M}(1)(\chi)$ has obviously a trivial action of $\operatorname{Ker}(\chi)$, showing $\mathscr{M}(1)(\chi) \subseteq\left(\mathscr{M}(1) \otimes R_{\chi}\right)^{\operatorname{Ker}(\chi)}$. Since, as the above examples show, we 
will be dealing only with functors verifying (3.1), this discussion enables us to replace $\Delta$ with $\Delta / \operatorname{Ker}(\chi) \cong \operatorname{Im}(\chi)$ without changing the $\chi$-components we are interested in. We can therefore assume that $p$ splits completely in $F$, and we assume this throughout.

Then we set

$$
U_{n}:=\left(\prod_{\mathrm{p} \mid p} \mathcal{O}_{F_{n}, \mathrm{p}}^{\times}\right)
$$

and we agree to let $\mathcal{O}_{n}^{\times}=\mathcal{O}_{F_{n}}^{\times}$and similarly $C y c_{n}=C y c_{F_{n}}$. Observe that since in a $\mathbb{Z}_{p^{-}}$ extension only primes above $p$ ramify, the set $\Sigma$ of Section 2 relative to $F_{n} / F$ is $\Sigma=\{\mathfrak{p} \subset$ $\left.\mathcal{O}_{F}, \mathfrak{p} \mid p\right\}$ for all $n$ and our notation is consistent with the one introduced there. We finally set

$$
B_{n}:=\left(\mathcal{O}_{n}^{\times} / C y c_{n}\right)(\chi), \quad \mathcal{B}_{n}:=\left(U_{n} / C y c_{n}\right)(\chi) \quad \text { and } \quad A_{n}:=C l_{F_{n}}(\chi) .
$$

By Sinnott's theorem above, together with [Sin81, theorem 5.3] that guarantees $p \nmid \kappa_{F_{n}}$ for all $n$, the groups $C l_{F_{n}} \otimes \mathbb{Z}_{p}$ and $\left(\mathcal{O}_{n}^{\times} / C y c_{n}\right) \otimes \mathbb{Z}_{p}$ have the same order. The characterby-character version of this result is much deeper: it is known as Gras' Conjecture, and is a consequence of the (now proven) Main Conjecture of Iwasawa Theory, as detailed in [Gre77] or [BNQD01]. It follows that $\left|A_{n}\right|=\left|B_{n}\right|$ for all $n \geqslant 0$.

Remark. The semi-local units considered by Gillard [Gil79b] are products over all $\mathfrak{p}$ above $p$ of local units that are $1(\bmod \mathfrak{p})$. In our situation, all completions $F_{\mathfrak{p}}$ at primes $\mathfrak{p} \mid p$ are isomorphic to $\mathbb{Q}_{p}$, so the two definitions coincide and $U_{n}(\chi)$ is a free $R_{\chi}$-module of rank 1.

Moreover, since $p$ splits completely in $F / \mathbb{Q}$, all primes above $p$ totally ramify in $F_{n} / F$ : let $\mathfrak{P}_{n}$ be one such a prime. Its image in $A_{n}$ generates a subgroup which is independent of the choice of $\mathfrak{P}_{n}$ : indeed, all primes above $p$ are conjugate by an element in $\Delta$, and the image in $A_{n}$ of $\delta\left(\mathfrak{P}_{n}\right)$ is $\mathfrak{P}_{n}^{\chi(\delta)}$, which generates the same subgroup as $\mathfrak{P}_{n}$. We call this subgroup $\Pi_{n}$ and we can restate Lemma $2 \cdot 1$ saying that $\operatorname{Im}(\beta)=\delta\left(\Pi_{n}\right)$ (use also Lemma 2.3).

We now investigate in some detail the structure of $C y c_{n}$. First of all, letting $f$ be the conductor of $F$, we define

$$
\eta_{n}:=\left(\operatorname{Norm}_{F_{n}}^{\mathbb{Q}\left(\zeta_{f p^{n+1}}\right)}\left(1-\zeta_{f p^{n+1}}\right)\right) \otimes\left(\sum_{\delta \in \Delta} \chi\left(\delta^{-1}\right) \delta\right) \in C y c_{n}(\chi) .
$$

The left factor is a unit since $p \nmid f$ because $p$ splits completely in $F$, it is cyclotomic by definition and is acted upon by $\Delta$ through $\chi$ : morever, Sinnott's description of cyclotomic units shows that $C y c_{n}(\chi)=\eta_{0} R_{\chi} \times \eta_{n} R_{\chi}\left[G_{n}\right]$ (see, for instance, [Gil79a, section 3] for details). In particular, we have an isomorphism of $G_{n}$-modules $C y c_{n}(\chi) \cong R_{\chi} \times I_{G_{n}}$ where $I_{G_{n}}$ is the augmentation ideal in $R_{\chi}\left[G_{n}\right]$, and we find a split exact sequence

$$
0 \longrightarrow\left\langle\eta_{n}\right\rangle \longrightarrow C y c_{n}(\chi) \longrightarrow\left\langle\eta_{0}\right\rangle \longrightarrow 0
$$

where we denote, here and in what follows, by $\left\langle\eta_{0}\right\rangle$ and $\left\langle\eta_{n}\right\rangle$ the $R_{\chi}\left[G_{n}\right]$-modules generated by $\eta_{0}$ and $\eta_{n}$ respectively: by the above isomorphisms, (3.2) corresponds to the sequence

$$
0 \longrightarrow I_{G_{n}} \longrightarrow I_{G_{n}} \times R_{\chi} \longrightarrow R_{\chi} \longrightarrow 0 .
$$

LEMMA 3.1. We have $\hat{H}^{0}\left(G_{n, m},\left\langle\eta_{n}\right\rangle\right)=0$ and $\hat{H}^{-1}\left(G_{n, m},\left\langle\eta_{0}\right\rangle\right)=0$. In particular, the natural map $\hat{H}^{q}\left(G_{n, m}, C y c_{n}(\chi)\right) \cong \hat{H}^{q}\left(G_{n, m},\left\langle\eta_{0}\right\rangle\right) \times \hat{H}^{q}\left(G_{n, m},\left\langle\eta_{n}\right\rangle\right)$ induced by (3.2) gives 
isomorphisms of $\Delta$-modules

$$
\hat{H}^{0}\left(G_{n, m}, C y c_{n}(\chi)\right) \cong \hat{H}^{0}\left(G_{n, m},\left\langle\eta_{0}\right\rangle\right)
$$

and

$$
\hat{H}^{-1}\left(G_{n, m}, C y c_{n}(\chi)\right) \cong \hat{H}^{-1}\left(G_{n, m},\left\langle\eta_{n}\right\rangle\right) .
$$

Both are groups of order $p^{d n}$ where $d=r k_{\mathbb{Z}_{p}} R_{\chi}$.

Proof. The exact sequence

$$
0 \longrightarrow I_{G_{n}} \longrightarrow R_{\chi}\left[G_{n}\right] \longrightarrow R_{\chi} \longrightarrow 0
$$

shows, since $\hat{H}^{q}\left(G_{n, m}, R_{\chi}\left[G_{n}\right]\right)=0$ for all $q$, that $\hat{H}^{0}\left(G_{n, m}, I_{G_{n}}\right) \cong \hat{H}^{-1}\left(G_{n, m}, R_{\chi}\right)=0$. The $R_{\chi}\left[G_{n}\right]$-isomorphisms $\left\langle\eta_{n}\right\rangle \cong I_{G_{n}}$ and $\left\langle\eta_{0}\right\rangle \cong R_{\chi}$ give the result.

Suppose now that $\lambda=0$. The extension $F_{\infty} / F$ is totally ramified since $p$ splits in $F$ and the maps $N_{m}^{n}: A_{n} \rightarrow A_{m}$ (induced by norms on $(\chi)$-parts, and that we keep on calling "Norm maps" by a slight abuse of notation) are surjective by class field theory for all $n \geqslant m$; assuming $\lambda=0$ and choosing $m$ big enough, the orders of $A_{n}$ and $A_{m}$ coincide, and these norm maps are actually isomorphisms. Therefore the projective limit $X=\lim A_{n}$ with respect to norms stabilizes to a finite group and $A_{n} \cong X$ for all $n \gg 0$ (we introduce here the notation $a \gg b$, equivalent to $b \ll a$, to mean that there exists a $b_{0} \geqslant b$ such that what we are stating holds for all $a \geqslant b_{0}$ ). In particular, the action of $\Gamma$ on $X$ must factor through a certain quotient $G_{m}=\Gamma / \Gamma^{p^{m}}$. Therefore $G_{n, m}$ acts trivially on $A_{n}$ for all $n \geqslant m$ and the $G_{n, m}$-norm $N_{G_{n, m}}=\sum_{\tau \in G_{n, m}} \tau$ acts on $A_{n}$ as multiplication by $p^{n-m}$. Choosing $n$ big enough so that $p^{n-m} A_{n}=0$, we find $N_{G_{n, m}} A_{n}=0$ and

$$
\begin{aligned}
\hat{H}^{0}\left(G_{n, m}, A_{n}\right) & =A_{n}^{G_{n, m}} / N_{G_{n, m}} A_{n}=A_{n} \\
& =A_{n}\left[N_{G_{n, m}}\right] / I_{G_{n, m}} A_{n}=\hat{H}^{-1}\left(G_{n, m}, A_{n}\right)
\end{aligned}
$$

where $I_{G_{n, m}}$ is the augmentation ideal of $R_{\chi}\left[G_{n, m}\right]$. Therefore $A_{n} \cong \hat{H}^{-1}\left(G_{n, m}, A_{n}\right) \cong$ $\hat{H}^{0}\left(G_{n, m}, A_{n}\right)$, whenever $\lambda=0$ and $n \gg m \gg 0$. A similar argument leads to the equivalent of (3.4) for $B_{n}$, namely $\hat{H}^{q}\left(G_{n, m}, B_{n}\right) \cong B_{n}$ for all $q \in \mathbb{Z}$.

LEMMA 3.2. If $\lambda=0$ and $m \gg 0$, the natural map

$$
H^{1}\left(G_{n, m}, C y c_{n}(\chi)\right) \longrightarrow H^{1}\left(G_{n, m}, \mathcal{O}_{n}^{\times}(\chi)\right)
$$

is injective for all $n \geqslant m$.

Proof. Taking $G_{n, m}$-cohomology in the exact sequence defining $B_{n}$ gives

$$
\begin{aligned}
0 \longrightarrow H^{0}\left(G_{n, m}, C y c_{n}(\chi)\right) \longrightarrow & H^{0}\left(G_{n, m}, \mathcal{O}_{n}^{\times}(\chi)\right) \longrightarrow H^{0}\left(G_{n, m}, B_{n}\right) \longrightarrow \\
& \longrightarrow H^{1}\left(G_{n, m}, C y c_{n}(\chi)\right) \longrightarrow H^{1}\left(G_{n, m}, \mathcal{O}_{n}^{\times}(\chi)\right) .
\end{aligned}
$$

Since $G_{n, m}$-invariants of $C y c_{n}(\chi)$ and $\mathcal{O}_{n}^{\times}(\chi)$ are $C y c_{m}(\chi)$ and $\mathcal{O}_{m}^{\times}(\chi)$ respectively, we immediately see that $\operatorname{Ker}\left(H^{1}\left(G_{n, m}, C y c_{n}(\chi)\right) \longrightarrow H^{1}\left(G_{n, m}, \mathcal{O}_{n}^{\times}(\chi)\right)\right)=B_{n}^{G_{n, m}} / B_{m}$. Assuming that $m$ is big enough and $\lambda=0$ implies that the orders of $B_{n}$ and $B_{m}$ coincide, and the same holds, a fortiori, for $B_{n}^{G_{n, m}}$ and $B_{m}$. Thus, the above kernel is trivial. 


\section{Semi-local units modulo cyclotomic units}

We now state a very useful result about semi-local units in our setting; it can already be found in a paper by Iwasawa [Iwa60]. We keep the same notation introduced in the previous section and we make from now on constant use of Lemma 2.3 above, especially in the form of the isomorphism in (2.2).

Definition 4.1. We define $U_{n}^{1}$ to be the kernel $U_{n}^{1}=\operatorname{Ker}\left(N_{0}^{n}: U_{n} \rightarrow U_{0}\right)$ and we set $\mathcal{B}_{n}^{1}=U_{n}^{1}(\chi) /\left\langle\eta_{n}\right\rangle$.

Proposition 4.2. The natural map $U_{n}^{1} \times U_{0} \hookrightarrow U_{n}$ induced by injections is an isomorphism of $\Delta \times G_{n}$-modules. It induces a decomposition $\mathcal{B}_{n} \cong \mathcal{B}_{n}^{1} \times \mathcal{B}_{0}$.

Proof. Since all primes above $p$ are totally ramified in $F_{n} / F$ we can restrict our attention to a single prime $\mathfrak{p} \mid p$ in $\mathcal{O}_{F}$ and show that

$$
\mathcal{O}_{n, \mathfrak{p}}^{\times 1} \times \mathcal{O}_{0, \mathfrak{p}}^{\times} \cong \mathcal{O}_{n, \mathfrak{p}}
$$

where we denote by $\mathcal{O}_{n, \mathfrak{p}}^{\times 1}$ the local units having norm 1 in $\mathcal{O}_{0, \mathfrak{p}}^{\times}$: taking the product over all primes above $p$ will give the first statement of the Proposition. Consider the exact sequence induced by the norm map $N_{0}^{n}$

$$
0 \longrightarrow \mathcal{O}_{n, \mathfrak{p}}^{\times 1} \longrightarrow \mathcal{O}_{n, \mathfrak{p}}^{\times} \longrightarrow N_{0}^{n}\left(\mathcal{O}_{n, \mathfrak{p}}^{\times}\right) \subseteq \mathcal{O}_{0, \mathfrak{p}}^{\times} \longrightarrow 0 .
$$

Since the extension $F_{n, \mathfrak{p}} / F_{0, \mathfrak{p}}$ is cyclic and totally ramified, local class field theory shows that $\hat{H}^{0}\left(G_{n}, \mathcal{O}_{n, \mathfrak{p}}^{\times}\right)=\mathcal{O}_{0, \mathfrak{p}}^{\times} / N_{0}^{n}\left(\mathcal{O}_{n, \mathfrak{p}}^{\times}\right)$is a cyclic group of order $p^{n}$. As $\mathcal{O}_{0, \mathfrak{p}}^{\times} \cong \mathbb{Z}_{p}^{\times}$this shows $N_{0}^{n}\left(\mathcal{O}_{n, \mathfrak{p}}^{\times}\right)=\left(\mathcal{O}_{0, \mathfrak{p}}^{\times}\right)^{p^{n}} \times \mu_{p-1}$ and since $\mathcal{O}_{0, \mathfrak{p}}^{\times}$contains no roots of unity of $p$-power order we can identify this group with $\mathcal{O}_{0, \mathfrak{p}}^{\times}$simply by extracting $p^{n}$ th roots. We find

$$
0 \longrightarrow \mathcal{O}_{n, \mathfrak{p}}^{\times 1} \longrightarrow \mathcal{O}_{n, \mathfrak{p}}^{\times} \stackrel{p^{n}}{\longrightarrow} \mathcal{O}_{0, \mathfrak{p}}^{\times} \longrightarrow 0 .
$$

Since the natural embedding $\mathcal{O}_{0, \mathrm{p}}^{\times} \hookrightarrow \mathcal{O}_{n, \mathrm{p}}^{\times}$is a $G_{n}$-linear section of (4-1), it splits the sequence and therefore gives an isomorphism $\mathcal{O}_{n, \mathfrak{p}}^{\times 1} \times \mathcal{O}_{0, \mathfrak{p}}^{\times} \cong \mathcal{O}_{n, \mathfrak{p}}$.

The explicit description $C y c_{n}(\chi)=\left\langle\eta_{0}\right\rangle \times\left\langle\eta_{n}\right\rangle$ together with $N_{0}^{n}\left(\eta_{n}\right)=0$ (see the discussion before equation (3.2)) shows that $N_{0}^{n}\left(C y c_{n}(\chi)\right)=N_{0}^{n}\left(\left\langle\eta_{0}\right\rangle\right)=\left\langle\eta_{0}^{p^{n}}\right\rangle$. The argument above thus gives the commutative diagram:

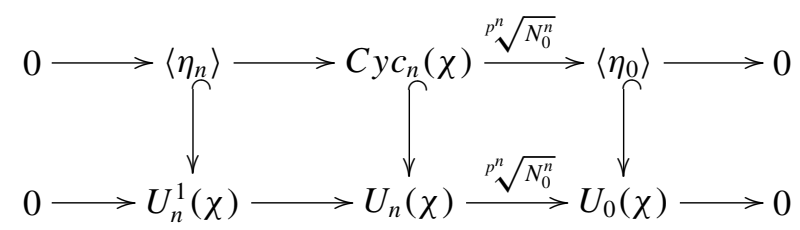

and this proves $\mathcal{B}_{n} \cong \mathcal{B}_{n}^{1} \times \mathcal{B}_{0}$.

More useful than the splitting itself is the following easy consequence:

COROLlaRY 4.3. For every $n \geqslant m \geqslant 0$ and for every $q \in \mathbb{Z}$ the natural maps

$$
\hat{H}^{q}\left(G_{n, m}, U_{n}(\chi)\right) \longrightarrow \hat{H}^{q}\left(G_{n, m}, U_{n}^{1}(\chi)\right) \times \hat{H}^{q}\left(G_{n, m}, U_{0}(\chi)\right)
$$

and

$$
\hat{H}^{q}\left(G_{n, m}, \mathcal{B}_{n}\right) \longrightarrow \hat{H}^{q}\left(G_{n, m}, \mathcal{B}_{n}^{1}\right) \times \hat{H}^{q}\left(G_{n, m}, \mathcal{B}_{0}\right)
$$


induced by Proposition 4.2 are isomorphisms of abelian groups. In particular, we have $\hat{H}^{-1}\left(G_{n, m}, U_{n}(\chi)\right)=\hat{H}^{-1}\left(G_{n, m}, U_{n}^{1}(\chi)\right)$ and $\hat{H}^{0}\left(G_{n, m}, U_{n}(\chi)\right)=\hat{H}^{0}\left(G_{n, m}, U_{0}(\chi)\right)$.

Proof. The splitting of the cohomology groups follows immediately from the Proposition. Concerning the cohomology of $U_{n}(\chi)$, we observe that, since $G_{n, m}$ acts trivially on the torsion-free module $U_{0}$, the group $\hat{H}^{0}\left(G_{n, m}, U_{0}\right)$ is a group of order $p^{[F: \mathbb{Q}](n-m)}$ while $\hat{H}^{-1}\left(G_{n, m}, U_{0}\right)=0$. This already implies that $\hat{H}^{-1}\left(G_{n, m}, U_{n}\right)=\hat{H}^{-1}\left(G_{n, m}, U_{n}^{1}\right)$. It also shows that $\hat{H}^{0}\left(G_{n, m}, U_{n}^{1}\right)$ must be trivial because $\hat{H}^{0}\left(G_{n, m}, U_{n}\right)$ has order $p^{[F: \mathbb{Q}](n-m)}$ by local class field theory; Lemma 2.3 then allows to take $(\chi)$-parts and to get the desired equalities.

LEMMA 4.4. For all $n \gg m \geqslant 0$, there are isomorphisms $\hat{H}^{q}\left(G_{n, m}, \mathcal{B}_{n}^{1}\right) \cong R_{\chi} / L_{p}(1, \chi)$ and $\hat{H}^{q}\left(G_{n, m}, \mathcal{B}_{0}\right) \cong R_{\chi} / L_{p}(1, \chi)$ holding for every $q \in \mathbb{Z}$.

Proof. Let $\Lambda:=R_{\chi}[[T]]$ and fix an isomorphism $\varpi: \Lambda \cong R_{\chi}[[\Gamma]]$. This isomorphism fixes a choice of a topological generator $\varpi(1+T)=: \gamma_{0}$ of $\Gamma$ and we denote by $\kappa \in \mathbb{Z}_{p}^{\times}$the element $\varepsilon_{c y c}\left(\gamma_{0}\right)$ where

$$
\varepsilon_{c y c}: \operatorname{Gal}(\bar{F} / F) \longrightarrow \mathbb{Z}_{p}^{\times}
$$

is the cyclotomic character of $F$. The main tool of the proof will be [Gil79b, theorem 2], that gives isomorphisms of $R_{\chi}[[\Gamma]]$-modules

$$
\mathcal{B}_{0} \cong R_{\chi} / L_{p}(1, \chi) \quad \text { and } \quad \mathcal{B}_{n} \cong \Lambda /\left(f(T), \omega_{n}(T) / T\right)
$$

where $\omega_{n}(T)=(1+T)^{p^{n}}-1$ and $f(T) \in \Lambda$ is the power series verifying $f\left(\kappa^{s}-1\right)=$ $L_{p}(1-s, \chi)$ for all $s \in \mathbb{Z}_{p}$. We make $\Gamma$ act on the modules appearing in (4.2) by $\gamma_{0} \cdot x=$ $\varpi^{-1}\left(\gamma_{0}\right) x=(1+T) x$ for all $x \in \mathcal{B}_{0}$ (resp. all $x \in \mathcal{B}_{n}$ ): this induces the action of $G_{n, m}$ we need to compute the cohomology with respect to.

Starting with $\mathcal{B}_{0}$, observe that the action of $\Gamma$, and thus of its subquotient $G_{n, m}$, is trivial on the finite group $\mathcal{B}_{0}$ : as in (3.4) we get

$$
\hat{H}^{q}\left(G_{n, m}, \mathcal{B}_{0}\right) \cong \mathcal{B}_{0} \quad \text { for all } n \gg m \geqslant 0,
$$

and we apply (4.2) to get our claim.

Now we compute $\hat{H}^{-1}\left(G_{n, m}, \mathcal{B}_{n}^{1}\right)$ : by definition, $\hat{H}^{-1}\left(G_{n, m}, \mathcal{B}_{n}^{1}\right)=\mathcal{B}_{n}^{1}\left[N_{G_{n, m}}\right] / I_{G_{n, m}} \mathcal{B}_{n}^{1}$. Applying $\varpi$ we find $I_{G_{n, m}} \cong \omega_{m}(T)\left(\Lambda / \omega_{n}(T)\right)$ and $\varpi\left(N_{G_{n, m}}\right)=v_{n, m}(T)$ where $v_{n, m}(T):=$ $\omega_{n}(T) / \omega_{m}(T)$. Hence

$$
\hat{H}^{-1}\left(G_{n, m}, \mathcal{B}_{n}^{1}\right) \cong \frac{\left\{g(T) \in \Lambda \mid g(T) v_{n, m}(T) \in\left(f(T), \omega_{n}(T) / T\right)\right\}}{\left(f(T), \omega_{n}(T) / T, \omega_{m}(T)\right)} .
$$

As observed in [Gil79b, lemma 5], $f(T)$ and $\omega_{n}(T) / T$ have no common zeroes. Therefore a relation

$$
g(T) \frac{\omega_{n}(T)}{\omega_{m}(T)}=a(T) f(T)+b(T) \frac{\omega_{n}(T)}{T}
$$

implies $v_{n, m}(T) \mid a(T)$ and we find $g(T)=c(T) f(T)+b(T) \omega_{m}(T) / T$ for some $c(T) \in \Lambda$ : thus,

$$
\begin{aligned}
\hat{H}^{-1}\left(G_{n, m}, \mathcal{B}_{n}^{1}\right) & \cong \frac{\left(f(T), \omega_{m}(T) / T\right)}{\left(f(T), \omega_{n}(T) / T, \omega_{m}(T)\right)} \\
& \cong \frac{\left(\omega_{m}(T) / T\right)}{\left(f(T), \omega_{n}(T) / T, \omega_{m}(T)\right)}
\end{aligned}
$$


The evaluation map $g(T) \omega_{m}(T) / T \mapsto g(0)$ gives an isomorphism

$$
\frac{\left(\omega_{m}(T) / T\right)}{\left(\omega_{m}(T)\right)} \cong R_{\chi}
$$

and we find

$$
\frac{\left(\omega_{m}(T) / T\right)}{\left(f(T), \omega_{n}(T) / T, \omega_{m}(T)\right)} \cong R_{\chi} /\left(f(0), \omega_{n}(0)\right) .
$$

Since this last module is $R_{\chi} / f(0)$ as soon as $n$ is big enough and, by definition, $f(0)=$ $L_{p}(1, \chi)$, we get our claim for $q=-1$. Using now that $\mathcal{B}_{n}^{1}$ is finite and therefore has a trivial Herbrand quotient, we know that the order of $\hat{H}^{0}\left(G_{n, m}, \mathcal{B}_{n}^{1}\right)$ is the predicted one: the fact that it is cyclic as an $R_{\chi}$-module comes from the exact sequence

$$
\begin{aligned}
0 \longrightarrow \hat{H}^{0}\left(G_{n, m}, \mathcal{B}_{n}^{1}\right) \longrightarrow \hat{H}^{0}\left(G_{n, m}, U_{n}^{1}(\chi)\right) \longrightarrow & \hat{H}^{0}\left(G_{n, m},\left\langle\eta_{n}\right\rangle\right) \longrightarrow \\
& \longrightarrow \hat{H}^{1}\left(G_{n, m}, \mathcal{B}_{n}^{1}\right) \longrightarrow 0
\end{aligned}
$$

since $\hat{H}^{0}\left(G_{n, m}, U_{n}^{1}(\chi)\right)$ is itself cyclic, as discussed in Corollary $4 \cdot 3$.

Finally, the fact that $G_{n, m}$ is cyclic gives isomorphisms in Tate cohomology $\hat{H}^{2 q}\left(G_{n, m}, M\right) \cong \hat{H}^{0}\left(G_{n, m}, M\right)$ for all modules $M$ (and analogously $\hat{H}^{2 q+1}\left(G_{n, m}, M\right) \cong$ $\left.\hat{H}^{-1}\left(G_{n, m}, M\right)\right)$, so the claim for all $q$ 's follows from our computation in the cases $q=0,-1$.

We want to stress that $R_{\chi}$ is an unramified extension of $\mathbb{Z}_{p}$. In particular, $p$ is a uniformizer in $R_{\chi}$ and therefore the $p$-adic valuation of an element in $R_{\chi}$ is an integer: for $a, b \in R_{\chi}$ we will write $a \stackrel{p}{=} b$ to mean that both sides have the same valuation.

Proposition 4.5. Recall that $X=\underline{\lim } A_{n}$ : if $\lambda=0$ then $\left|X^{\Gamma}\right| \stackrel{p}{=} L_{p}(1, \chi)^{d}$ where $d=r k_{\mathbb{Z}_{p}} R_{\chi}$.

Proof. Let $L_{0}$ be the maximal pro- $p$ abelian extension of $F_{\infty}$ everywhere unramified and let $M_{0}$ be the maximal pro- $p$ abelian extension of $F_{\infty}$ unramified outside $p$. We claim that $L_{0}=M_{0}$. This follows from the fact that for every $\mathfrak{p} \subseteq \mathcal{O}_{F}$ dividing $p$, the local field $F_{\mathfrak{p}}$ is $\mathbb{Q}_{p}$, since $p$ splits completely, and it therefore admits only two independent $\mathbb{Z}_{p}$-extensions by local class field theory. In particular, every pro- $p$ extension of $F_{\infty, \mathfrak{p}}$ that is abelian over $F_{\mathfrak{p}}$ must be unramified, so $M_{0}=L_{0}$. Now let $Y:=\operatorname{Gal}\left(L_{\infty} / F_{\infty}\right)$ where $L_{\infty}$ is the maximal pro- $p$ abelian extension of $F_{\infty}$ everywhere unramified: then the Artin reciprocity map gives an isomorphism $X \cong Y(\chi)$; also, let $M_{\infty}$ be the maximal pro- $p$ abelian extension of $F_{\infty}$ unramified outside $p$ and $\mathscr{Y}:=\operatorname{Gal}\left(M_{\infty} / F_{\infty}\right)$. A classical argument (see [Was97, chapter 13]) shows that $Y_{\Gamma}=\operatorname{Gal}\left(L_{0} / F_{\infty}\right)$ and $\mathscr{Y}_{\Gamma}=\operatorname{Gal}\left(M_{0} / F_{\infty}\right)$ : our claim above implies that $Y_{\Gamma}=\mathscr{Y}_{\Gamma}$. Since the actions of $\Delta$ and $\Gamma$ commute with each other, this also shows $X_{\Gamma}=$ $\mathscr{Y}(\chi)_{\Gamma}$. Combine this with the following exact sequence induced by multiplication by $\gamma_{0}-1$ where $\gamma_{0}$ is a topological generator of $\Gamma$

$$
0 \longrightarrow X^{\Gamma} \longrightarrow X \stackrel{\gamma_{0}-1}{\longrightarrow} X \longrightarrow X_{\Gamma} \longrightarrow 0:
$$

since we assumed $\lambda=0$ all the groups are finite and the sequence gives $\left|X^{\Gamma}\right|=$ $\left|X_{\Gamma}\right|=\left|\mathscr{Y}(\chi)_{\Gamma}\right|$. The Main Conjecture of Iwasawa Theory, as proved by Rubin of [Lan90, appendix], shows that the characteristic polynomial of $\mathscr{Y}(\chi)$ is $f(T)$ (see the proof of Lemma 4.4). Since $\mathscr{Y}$ contains no non-zero finite $\Gamma$-submodules (see [NSW00]), we find $\mathscr{Y}^{\Gamma}=0$ and the order of $\mathscr{Y}(\chi)_{\Gamma}$ is $f(0)^{d} \stackrel{p}{=} L_{p}(1, \chi)^{d}$, as detailed in [Was97, exercise 13.12]. 
COROLlaRY 4.6. If $\lambda=0$, then $\Pi_{n}$ is a cyclic $R_{\chi}$-module of order $\left|L_{p}(1, \chi)\right|_{p}^{-d}$ for every $n \gg 0$.

Proof. Indeed, [Gre76, theorem 2] shows that $\lambda=0$ if and only if $X^{\Gamma}=\Pi_{n}$. The result now follows from the proposition and from the remark of Section 3.

\section{Main result}

We are now in position of proving our main result. We stick to the notation introduced in Section 3. Let $n \geqslant 0$ and let $Q_{n}:=\left(\mathbb{U}_{F_{n}} / \mathcal{O}_{n}^{\times}\right)(\chi)$ as in Section 2: consider the exact sequence

$$
0 \longrightarrow \mathcal{O}_{n}^{\times}(\chi) \longrightarrow \mathbb{U}_{F_{n}}(\chi) \longrightarrow Q_{n} \longrightarrow 0 .
$$

Since $C y c_{n}(\chi) \subseteq \mathcal{O}_{n}^{\times}(\chi)$, it induces an exact sequence

$$
0 \longrightarrow B_{n} \longrightarrow\left(\mathbb{U}_{F_{n}} / C y c_{n}\right)(\chi) \longrightarrow Q_{n} \longrightarrow 0,
$$

and the Tate cohomology of $\left(\mathbb{U}_{F_{n}} / C y c_{n}\right)(\chi)$ coincides with that of $\mathcal{B}_{n}$, as discussed in Section 2 . For every $m \leqslant n$ the cyclicity of Tate cohomology for cyclic groups induces an exact square

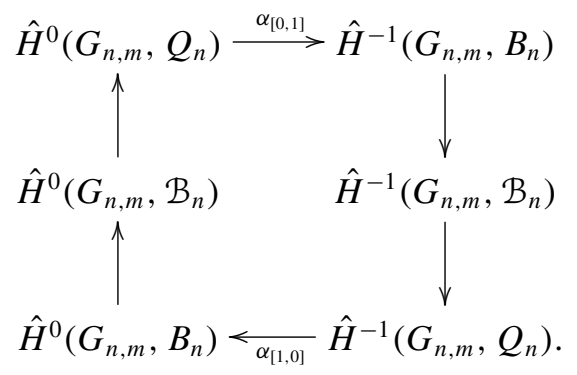

Consider now the exact sequence

$$
0 \longrightarrow Q_{n} \longrightarrow C_{F_{n}}(\chi) \longrightarrow A_{n} \longrightarrow 0:
$$

by Lemma 2.3 , we have $\hat{H}^{q}\left(G_{n, m}, C_{n}(\chi)\right) \cong \hat{H}^{q}\left(G_{n, m}, C_{n}\right)(\chi)$ for all $q \in \mathbb{Z}$; global class field theory (see section 11.3 of Tate's paper in [CF86]) shows $\hat{H}^{q}\left(G_{n, m}, C_{n}\right)(\chi) \cong$ $\hat{H}^{q+2}\left(G_{n, m}, \mathbb{Z}(\chi)\right)=0$ because we assumed $\chi \neq 1$. Therefore the long exact cohomology sequence of (5.2) induces isomorphisms

$$
\hat{H}^{q}\left(G_{n, m}, A_{n}\right) \cong \hat{H}^{q+1}\left(G_{n, m}, Q_{n}\right) \text { for every } q \in \mathbb{Z} .
$$

Remark. Observe that our discussion never uses the assumption $\chi(p)=1$. Indeed, the maps $\alpha_{[0,1]}$ and $\alpha_{[1,0]}$ are defined whenever $\chi \neq 1$ and their composition with the isomorphisms in (5.3) are indeed the same maps appearing in [KS95, proposition 2.6], where the case $\chi(p) \neq 1$ is treated. As discussed in the introduction, in that case they turned out to be isomorphisms if $\lambda=0$ (see also [BNQD01], [Kuz96] and [Oza97]). We are going to see this is not the case if $\chi(p)=1$. Before stating the next theorem we recall that $R_{\chi}=\mathbb{Z}_{p}[\operatorname{Im}(\chi)]$ with $\Delta$-action $\delta \cdot x=\chi(\delta) x$ for all $\delta \in \Delta$ : its rank over $\mathbb{Z}_{p}$ is denoted by $d$.

THEOREM 5.1. Let $F$ be a real abelian field such that $p \nmid[F: \mathbb{Q}]$ and fix a non-trivial character $\chi$ of $\Delta$ such that $\chi(p)=1$. Assume that $\lambda=0$ and let $n \gg m \gg 0$ be big enough. Then the kernels $\operatorname{Ker}\left(\alpha_{[0,1]}\right), \operatorname{Ker}\left(\alpha_{[1,0]}\right)$ and the cokernels $\operatorname{Coker}\left(\alpha_{[0,1]}\right), \operatorname{Coker}\left(\alpha_{[1,0]}\right)$ of the maps introduced in (5.1) are cyclic $R_{\chi}$-modules of order $\left|L_{p}(1, \chi)\right|_{p}^{-d}$. 
Proof. We start by determining $\operatorname{Ker}\left(\alpha_{[0,1]}\right)$. Choose $m$ big enough so that $\left|A_{m+k}\right|=\left|A_{m}\right|$ for all $k \geqslant 0$ and $n \geqslant m$ big enough so that $\hat{H}^{q}\left(G_{n, m}, A_{n}\right)=A_{n}$. As [Gre76, remark of section 2, proposition 2] shows that $\lambda=0$ implies $A_{m}=\operatorname{Ker}\left(J_{m, n}\right)$ if $n$ is sufficiently large. Combining Proposition $2 \cdot 2$ with (5.3), this gives an injection (here and in the rest of the proof we make constant use of Lemma 2.3)

$$
\hat{H}^{0}\left(G_{n, m}, Q_{n}\right) \longleftrightarrow \hat{H}^{-1}\left(G_{n, m}, \mathcal{O}_{n}^{\times}(\chi)\right) .
$$

Consider now the following commutative diagram, whose row and column are exact and where the injectivity of the vertical arrow in the middle follows from Lemma 3.2:

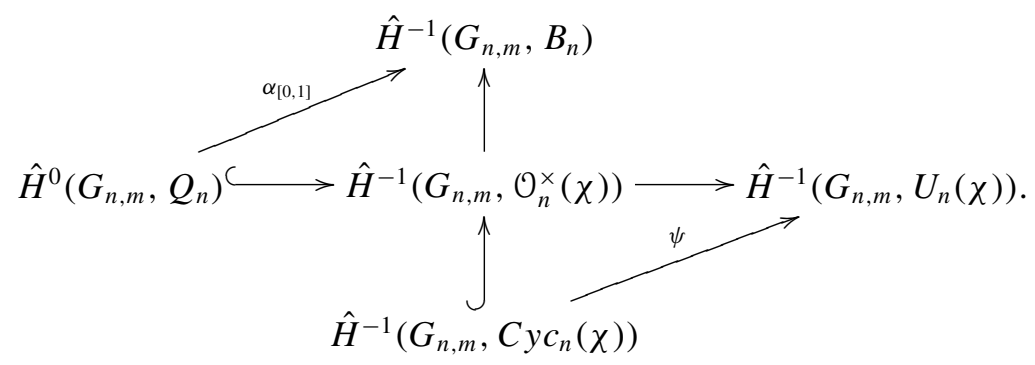

An easy diagram chase shows that $\operatorname{Ker}\left(\alpha_{[0,1]}\right) \cong \operatorname{Ker}(\psi)$. In order to study $\operatorname{Ker}(\psi)$, observe that $\psi$ appears in the sequence

$$
0 \longrightarrow \hat{H}^{0}\left(G_{n, m}, \mathcal{B}_{n}^{1}\right) \longrightarrow \hat{H}^{-1}\left(G_{n, m},\left\langle\eta_{n}\right\rangle\right) \stackrel{\psi}{\longrightarrow} \hat{H}^{-1}\left(G_{n, m}, U_{n}^{1}(\chi)\right),
$$

since $\hat{H}^{-1}\left(G_{n, m}, C y c_{n}(\chi)\right)=\hat{H}^{-1}\left(G_{n, m},\left\langle\eta_{n}\right\rangle\right)$ by Lemma $3 \cdot 1$ and $\hat{H}^{-1}\left(G_{n, m}, U_{n}(\chi)\right)=$ $\hat{H}^{-1}\left(G_{n, m}, U_{n}^{1}(\chi)\right)$ by Corollary 4.3. Morever, again by Corollary $4 \cdot 3, \hat{H}^{0}\left(G_{n, m}, U_{n}^{1}(\chi)\right)=$ 0 and (5.6) is exact, thus giving

$$
\operatorname{Ker}\left(\alpha_{[0,1]}\right) \cong \hat{H}^{0}\left(G_{n, m}, \mathcal{B}_{n}^{1}\right) \cong R_{\chi} / L_{p}(1, \chi),
$$

the last isomorphism being Lemma 4.4.

Having determined $\operatorname{Ker}\left(\alpha_{[0,1]}\right)$, the exactness of (5.1) together with Corollary $4 \cdot 3$ (and Lemma 4.4) show immediately that

$$
\operatorname{Coker}\left(\alpha_{[1,0]}\right) \cong \hat{H}^{0}\left(G_{n, m}, \mathcal{B}_{0}\right) \cong R_{\chi} / L_{p}(1, \chi) .
$$

Since the orders of $A_{n}$ and $B_{n}$ coincide for $n \gg 0$, and since these groups are isomorphic to $\hat{H}^{q}\left(G_{n, m}, Q_{n}\right)$ and $\hat{H}^{q}\left(G_{n, m}, B_{n}\right)$ respectively (see (3.4) and (5.3)), the equalities of orders

$$
\left|\operatorname{Ker}\left(\alpha_{[0,1]}\right)\right|=\left|\operatorname{Coker}\left(\alpha_{[0,1]}\right)\right| \text { and }\left|\operatorname{Ker}\left(\alpha_{[1,0]}\right)\right|=\left|\operatorname{Coker}\left(\alpha_{[1,0]}\right)\right|
$$

hold. By (5.7) and (5.8), the four groups have the same order, equal to $\left|L_{p}(1, \chi)\right|_{p}^{-d}$.

We are left with the structure of $\operatorname{Ker}\left(\alpha_{[1,0]}\right)$ and $\operatorname{Coker}\left(\alpha_{[0,1]}\right)$. The map $\alpha_{[1,0]}$ is the composition

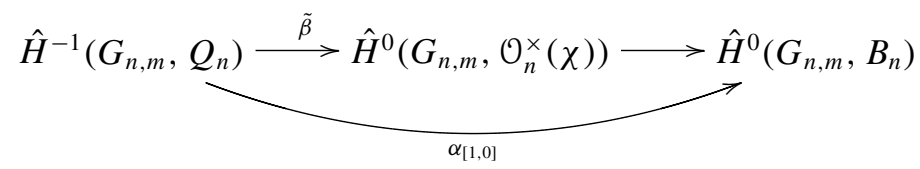

and $\operatorname{Ker}\left(\alpha_{[1,0]}\right) \supseteq \operatorname{Ker}(\tilde{\beta})=\Pi_{n}$, the last identification coming from Lemma $2 \cdot 1$ as discussed in the remark of Section 3. Combining Corollary 4.6 with the computation of the order of $\operatorname{Ker}\left(\alpha_{[1,0]}\right)$ performed above, the inclusion cannot be strict, and $\operatorname{Ker}\left(\alpha_{[1,0]}\right)$ is cyclic 
over $R_{\chi}$ of the prescribed order. Looking at $\operatorname{Ker}\left(\alpha_{[1,0]}\right)$ and at $\operatorname{Coker}\left(\alpha_{[0,1]}\right)$ as subgroups of $\hat{H}^{-1}\left(G_{n, m}, \mathcal{B}_{n}\right)$ as in $(5 \cdot 1)$, and knowing the structure of this last module by Lemma $4 \cdot 3$, shows that $\operatorname{Coker}\left(\alpha_{[0,1]}\right)$ is cyclic, too.

Now we can single out from the proof a precise description of the kernels of the maps $\alpha_{[1,0]}$ and $\alpha_{[0,1]}$ when seen as maps

$$
\alpha_{[i, j]}: A_{n} \longrightarrow B_{n},
$$

by combining (3.4) and (5.3). Observe that, by Lemma $(3 \cdot 1)$, there is an isomorphism $\hat{H}^{-1}\left(G_{n, m}, C y c_{n}(\chi)\right) \cong \hat{H}^{-1}\left(G_{n, m},\left\langle\eta_{n}\right\rangle\right)$, while $\hat{H}^{-1}\left(G_{n, m},\left\langle\eta_{n}\right\rangle\right) \cong \hat{H}^{-1}\left(G_{n, m}, I_{G_{n}}\right) \cong$ $\hat{H}^{0}\left(G_{n, m}, R_{\chi}\right)$ by (3.2) and (3.3). It is clear that these isomorphisms are not only $G_{n, m}$-linear, but also $G_{n}$-linear and therefore that $\hat{H}^{-1}\left(G_{n, m}, C y c_{n}(\chi)\right)$ has trivial $G_{n}$-action.

COROLLARY 5.2. With the same hypothesis as in the theorem,

$$
\operatorname{Ker}\left(\alpha_{[0,1]}\right)=\operatorname{Ker}\left(\alpha_{[1,0]}\right)=\Pi_{n},
$$

where $\Pi_{n}$ is the subgroup generated in $A_{n}$ by the class of any prime of $F_{n}$ above $p$, as discussed in the remark of Section 3.

Proof. While proving the theorem we found $\operatorname{Ker}\left(\alpha_{[1,0]}\right)=\Pi_{n}$, and we now focus on $\operatorname{Ker}\left(\alpha_{[0,1]}\right)$. Looking again at (5.5) we find $\operatorname{Ker}\left(\alpha_{[0,1]}\right) \subseteq \operatorname{Im}\left(\hat{H}^{-1}\left(G_{n, m}, C y c_{n}(\chi)\right)\right) \subseteq$ $\hat{H}^{-1}\left(G_{n, m}, \mathcal{O}_{n}^{\times}(\chi)\right)^{G_{n}}$ : since the isomorphisms $\hat{H}^{0}\left(G_{n, m}, Q_{n}\right) \cong A_{n}$ are $G_{n}$-linear, we get $\operatorname{Ker}\left(\alpha_{[0,1]}\right) \subseteq A_{n}^{G_{n}}$. As in the proof of Corollary (4.6), the assumption $\lambda=0$ is equivalent to $\Pi_{n}=X^{\Gamma}$ and $X^{\Gamma}=A_{n}^{G_{n}}$ if $n$ is big enough; putting all together, we have $\operatorname{Ker}\left(\alpha_{[0,1]}\right) \subseteq \Pi_{n}$. Since they have the same order thanks to Corollary 4.6 together with Theorem $5 \cdot 1$, the inclusion turns into an equality.

Remark. As the above Corollary shows, there are indeed two maps $\alpha_{[0,1]}$ and $\alpha_{[1,0]}$ sitting in an exact sequence

$$
0 \longrightarrow \Pi_{n} \longrightarrow A_{n} \stackrel{\alpha}{\rightarrow} B_{n} \longrightarrow \mathcal{B}_{0} / \eta_{0} \longrightarrow 0,
$$

where $\alpha$ can be either of them. This is the same as in the non-split case, where both $\alpha_{[0,1]}$ and $\alpha_{[1,0]}$ give an isomorphism $A_{n} \cong B_{n}$ for $n \gg 0$ if $\lambda=0$ (see [KS95]).

Acknowledgements. This work is part of my PhD thesis, written under the supervision of René Schoof. I would like to take this opportunity to thank him not only for proposing me to work on this subject and for the help he gave me in writing this paper, but especially for all the time and patience he put in following me through my $\mathrm{PhD}$ and for the viewpoint on Mathematics he suggested to me.

It is also a pleasure to thank the anonymous referee for many useful corrections and suggestions that improved the style and the mathematics of this text.

\section{REFERENCES}

[BNQD01] J.-R. BelLiARd and T. NGUYEn QUANG Do. Formules de classes pour les corps abéliens réels. Ann. Inst. Fourier (Grenoble) 51 (2001), no. 4, 903-937.

[CF86] J. W. S. CASSELS and A. FRÖHLICH (eds.). Algebraic number theory. (Academic Press Inc. [Harcourt Brace Jovanovich Publishers], 1986.) Reprint of the 1967 original.

[FW79] B. FERRERO and L. C. WASHINGTON. The Iwasawa invariant $\mu_{p}$ vanishes for abelian number fields. Ann. of Math. (2) 109 (1979), no. 2, 377-395.

[Gil79a] R. GILlaRD. Remarques sur les unités cyclotomiques et les unités elliptiques. J. Number Theory 11 (1979), no. 1, 21-48. 
[Gil79b] R. GiLlaRD. Unités cyclotomiques, unités semi-locales et $\mathbf{Z}_{l}$-extensions, II. Ann. Inst. Fourier (Grenoble) 29 (1979), no. 4, viii, 1-15.

[Gre76] R. GReEnberg. On the Iwasawa invariants of totally real number fields. Amer. J. Math. 98 (1976), no. 1, 263-284.

[Gre77] R. GreenBerg. On p-adic L-functions and cyclotomic fields, II. Nagoya Math. J. 67 (1977), 139-158.

[Iwa60] K. IWASAWA. On local cyclotomic fields. J. Math. Soc. Japan 12 (1960), 16-21.

[Iwa73] K. IWASAWA. On $\mathbf{Z}_{l}$-extensions of algebraic number fields. Ann. of Math. (2) 98 (1973), 246326.

[KS95] J. S. KRAFT and R. SCHOOF. Computing Iwasawa modules of real quadratic number fields. Compositio Math. 97 (1995), no. 1-2, 135-155, Special issue in honour of Frans Oort.

[Kuz96] L. V. KUZ'MIN. On formulas for the class number of real abelian fields. Izv. Ross. Akad. Nauk Ser. Mat. 60 (1996), no. 4, 43-110.

[Lan90] S. LANG. Cyclotomic fields I and II, second ed., Graduate Texts in Mathematics, vol. 121. (Springer-Verlag, 1990) With an appendix by Karl Rubin.

[NSW00] J. NEUKIRCH, A. SCHMIDT and K. WINGBERG. Cohomology of number fields, Grundlehren der Mathematischen Wissenschaften [Fundamental Principles of Mathematical Sciences], vol. 323, (Springer-Verlag, 2000).

[Oza97] M. OzAKI. On the cyclotomic unit group and the ideal class group of a real abelian number field, I, II. J. Number Theory 64 (1997), no. 2, 211-222, 223-232.

[Sch88] R. SchOOF. Cohomology of class groups of cyclotomic fields: an application to MorseSmale diffeomorphisms. J. Pure Appl. Algebra 53 (1988), no. 1-2, 125-137. MR MR955614 (89j:11111)

[Sin81] W. SinnotT. On the Stickelberger ideal and the circular units of an abelian field. Invent. Math. 62 (1980/81), no. 2, 181-234.

[Was97] L. C. WASHington. Introduction to cyclotomic fields, second ed. Graduate Texts in Mathematics, vol. 83 (Springer-Verlag, 1997). 